\title{
A STUDY OFं THE GALACTIC CENTRE REgION USING MIRA VARIABles
}

M.W. Feast

South African Astronomical Observatory, Cape, S.A.

Mira variables are attractive for galactic centre studies because (1) they are numerous there; (2) they have high luminosities ( $\mathrm{M}_{\mathrm{K}}$ ? -7.5) in the infrared where they can be seen through heavy absorption; (3) good radial velocities can be obtained from emission lines even for very faint objects; (4) at least in the solar neighbourhood and in globular clusters the period seems a good indicator of age and/or chemical composition.

Recent SAAO work covers four inter-related fields.

1. 2200 sets of JHKL $(1.2-3.5 \mu)$ measures of 220 nearby Miras have been made to establish their basic infrared properties (Feast, Catchpole, Robertson, Carter, Lloyd Evans). Me Miras occupy a limited region in the $\mathrm{J}-\mathrm{H} / \mathrm{H}-\mathrm{K}$ diagram allowing reddenings to be determined for those in the galactic centre.

2. Photographic work in $V$ and $I$ has been done (Lloyd Evans) in the galactic centre windows NGC $6522 / \mathrm{Sgr}$ I/Sgr II. A wide range of periods is present. Lack of long periods in previous work was due to a selection effect.

3. JHKL photometry of Miras in the windows (Glass) has begun. Scattered observations of 40 stars shows (a) $A_{K}=0.14 \pm .02\left(A_{V}=1.62\right.$ $\pm .17)$ for the NGC 6522 field and $A_{K}=0.11 \pm .01\left(A_{V}=1.25 \pm .15\right)$ for the Sgr I field; (b) a preliminary absolute magnitude calibration gives a mean distance modulus of $14.54(8.1 \mathrm{kpc})$. The s.e. of one observation $(\sim 0.5)$ should be much reduced when full light curves are available.

4. Radial velocities of Miras in the windows have been obtained (Feast). From 23 stars $(\bar{P}=223$ days $)$ the dispersion is $113 \mathrm{~km} / \mathrm{sec}$. This is the same as that of $\mathrm{OH} / \mathrm{IR}$ sources in the centre (Baud). These are usually considered as long period (young) Miras. Either (1) OH/IR Miras in the centre have shorter periods than elsewhere or (2) the kinematics of objects in the centre is independent of age or (3) the period-age relation in the solar neighbourhood does not apply in the central region.

The work is extended to include searches for heavily reddened Miras near the centre from which estimates can be made of the density distribution.

$$
376
$$

W. B. Burton (ed.). The Large-Scale (haracteristics of the Galaxy, 376.

Copyright $\odot 1979$ by the IAU. 